\title{
Port-Site Metastasis of Mucinous Borderline Ovarian Tumor after Laparoscopy
}

\author{
Naoto Furukawa ${ }^{a}$ Kazuhiro Nishioka ${ }^{b}$ Taketoshi Noguchi ${ }^{b}$ \\ Hirotaka Kajihara $^{b}$ Kiyoshige Horie ${ }^{b}$ \\ Departments of Obstetrics and Gynecology at ${ }^{a}$ Nara Medical University, Kashihara, and \\ ${ }^{b}$ Yamato Takada Municipal Hospital, Yamato Takada, Japan
}

\section{Key Words}

Borderline ovarian tumors · Port-site metastasis · Laparoscopy

\begin{abstract}
We report a case of port-site metastasis after laparoscopic surgery for borderline mucinous ovarian tumors (mBOTs) without spillage and review the related literature. The patient was a 50-year-old nulligravida who presented with abdominal distension. Magnetic resonance imaging showed a $20 \times 10-\mathrm{cm}$ multilocular mass with various signal intensities. The wall and septa of the mass were neither thick nor enhanced. A laparoscopy was performed. An intact left ovarian tumor was observed. The weight of the tumor was $1,540 \mathrm{~g}$. The final diagnosis was stage IA intestinal-type mBOT, so the patient did not undergo adjuvant therapy. Twentysix months after surgery, the patient presented with a $3 \times 5-\mathrm{cm}$ palpable mass on the umbilicus. Biopsy of the mass revealed mucinous adenocarcinoma and computed tomography showed a $3.5 \times 4.0-\mathrm{cm}$ mass at the umbilicus without additional metastases. A laparotomy was performed and no metastasis in the peritoneal cavity was observed by gross examination. An umbilical mass resection, hysterectomy, right salpingo-oophorectomy, appendectomy, and partial omentectomy were performed. Hematoxylin and eosin-stained sections of the umbilical mass revealed glands of varying size infiltrating the stroma, immunohistologic staining for cytokeratin 7 was positive, and cytokeratin 20 was negative, but no other metastases were observed. The patient was diagnosed with port-site metastasis and invasive recurrence of mBOT. She underwent six cycles of adjuvant paclitaxel and carboplatin therapy. Large ovarian tumors should be carefully extracted without spillage of the tumor contents to prevent port-site metastasis, despite the low incidence.
\end{abstract}


Furukawa et al.: Port-Site Metastasis of Mucinous Borderline Ovarian Tumor after Laparoscopy

\section{Introduction}

Minimally invasive surgery is a standard procedure for removing benign ovarian tumors, and it has become increasingly applied for the management of patients with mucinous ovarian tumors. Mucinous ovarian tumors are large, particularly among the intestinal type, and $15 \%$ of mucinous ovarian tumors are borderline mucinous ovarian tumors (mBOTs). Careful removal of the tumors without spillage of the cyst contents is crucial during surgery, particularly laparoscopy, to prevent metastasis. Although port-site metastasis is well known, it has a very low incidence. Here, we report a case of port-site metastasis after laparoscopic surgery for mBOTs without spillage and review the related literature.

\section{Case Presentation}

The patient was a 50-year-old nulligravida who presented with abdominal distension. Ultrasonography revealed a multilocular cyst in the pelvic cavity. Magnetic resonance imaging showed a $20 \times 10-\mathrm{cm}$ multilocular mass with various signal intensities. The wall and septa of the mass were neither thick nor enhanced. A laparoscopy was performed with insertion of a $10-\mathrm{mm}$ trocar through a small incision in the umbilicus and placement of two 5 - $\mathrm{mm}$ trocars $2 \mathrm{~cm}$ medial to the right anterior superior iliac spine and midline, $10 \mathrm{~cm}$ below the umbilical puncture. An intact left ovarian tumor was observed. Ascites was not observed. Peritoneal washing cytology was performed with negative findings. The infundibulopelvic ligament, ovarian ligament, and tube were sealed using a vessel sealing device and dissected. A Lap Disk Mini, protecting the incision area, was attached to the umbilical incision, which was widened to $2 \mathrm{~cm}$. The tumor was reduced by aspiration of the cyst contents (total 500 $\mathrm{ml}$ ) through the umbilical incision and removed through the umbilical incision without a retrieval bag. The weight of the tumor was $1,540 \mathrm{~g}$. Hematoxylin and eosin-stained sections revealed atypical epithelium-containing goblet cells that resembled gastrointestinal epithelium, but stromal invasion was negative (fig. 1). The final diagnosis was stage IA intestinal-type mBOT, so the patient did not undergo adjuvant therapy. Twenty-six months after surgery, the patient presented with a $3 \times 5-\mathrm{cm}$ palpable mass on the umbilicus. Biopsy of the mass revealed mucinous adenocarcinoma and computed tomography revealed a $3.5 \times$ 4.0-cm mass at the umbilicus without additional metastases (fig. 2). A laparotomy was performed and no metastasis in the peritoneal cavity was observed by gross examination. An umbilical mass resection, hysterectomy, right salpingo-oophorectomy, appendectomy, and partial omentectomy were performed. Hematoxylin and eosin-stained sections of the umbilical mass revealed glands of varying size infiltrating the stroma, immunohistologic staining for cytokeratin 7 was positive, and cytokeratin 20 was negative, but no other metastases were observed (fig. 3). The patient was diagnosed with port-site metastasis and invasive recurrence of mBOT. The patient underwent six cycles of adjuvant paclitaxel and carboplatin therapy.

\section{Discussion}

Benign ovarian tumors are usually resected laparoscopically. Mucinous ovarian tumors are large, however, and mBOTs are sometimes found after surgery even if mucinous ovarian tumors are suspected to be benign based on imaging. In the present case, magnetic resonance imaging did not suggest malignancy. The mean of 97 loculi in mBOTs is much 
Furukawa et al.: Port-Site Metastasis of Mucinous Borderline Ovarian Tumor after Laparoscopy

greater than the mean of 18 loculi found in benign mucinous ovarian tumors [1]. High signal intensity on T1-weighted image generally indicates hemorrhages or mucoid fluid and is visible in $52 \%$ of mBOTs [2]. The diagnostic criteria for mBOTs remain unclear. Large cysts increase the risk of rupture. Rupture of the tumor during surgery may lead to metastasis when the tumor is borderline malignant. Therefore, management of large ovarian tumors through laparotomy is preferred over laparoscopy to prevent metastasis due to rupture. A study comparing laparoscopy and laparotomy for borderline ovarian tumors [3] reported a similar incidence of metastasis between groups, but the mean tumor size was larger in the laparotomy group than in the laparoscopy group (13.5 vs. $7.5 \mathrm{~cm}$, respectively), indicating that surgeons tend to select laparotomy for large tumors. To prevent spillage of the tumor contents with laparoscopy, the tumor contents should be aspirated with a laparoscopic needle, avoiding accidental penetration through the tumor, and retrieved using a bag. It is difficult to reduce mBOTs compared with serous BOTs due to the multilocularity and the high viscosity of the mBOT cyst content. When the tumor is too large to be retrieved in a bag, it should be carefully extracted through the umbilical incision without contamination by the tumor contents. In the present case, however, port-site metastasis occurred despite careful extraction of the tumor. Contamination of the forceps or laparoscopic needle might also lead to metastasis. Another measure aimed at reducing port-site metastasis is irrigation of the trocar site with sterile water or $5 \%$ povidone iodine [4]. This procedure was not used in the present case. Port-site metastasis is an uncommon complication, occurring in $1 \%$ of laparoscopic surgeries for gynecologic malignancies [5, 6]. Furthermore, there are few reports of port-site metastasis of BOTs [3], so the incidence of port-site metastasis appears to be rarer for BOT than for ovarian cancer. The risk of progression to invasive carcinoma is very low in patients with early-stage BOTs $[7,8]$. A recent report discussed a mucinous subgroup of BOT in a small 'high-risk' group that is likely to recur invasively after fertilitysparing surgery [9].

When performing surgery for large ovarian tumors that are thought to be benign mucinous ovarian tumors based on magnetic resonance imaging, surgeons should consider the possibility of borderline malignancy. Large ovarian tumors should be carefully extracted without spillage of the tumor contents to prevent port-site metastasis, despite the low incidence. In these cases, surgeons should consider laparotomy rather than laparoscopy.

\section{Disclosure Statement}

The authors have no conflicts of interest to disclose.

\section{References}

1 Okamoto Y, Tanaka YO, Tsunoda H, Yoshikawa H, Minami M: Malignant or borderline mucinous cystic neoplasms have a larger number of loculi than mucinous cystadenoma: a retrospective study with MR. J Magn Reson Imaging 2007;26:94-99.

-2 Bent CL, Sahdev A, Rockall AG, Singh N, Sohaib SA, Reznek RH: MRI appearances of borderline ovarian tumours. Clin Radiol 2009;64:430-438.

-3 Fauvet R, Boccara J, Dufournet C, Poncelet C, Darai E: Laparoscopic management of borderline ovarian tumors: results of a French multicenter study. Ann Oncol 2005;16:403-410.

4 Ramirez PT, Wolf JK, Levenback C: Laparoscopic port-site metastases: etiology and prevention. Gynecol Oncol 2003;91:179-189.

5 Abu-Rustum NR, Rhee EH, Chi DS, Sonoda Y, Gemignani M, Barakat RR: Subcutaneous tumor implantation after laparoscopic procedures in women with malignant disease. Obstet Gynecol 2004;103:480-487. 


\section{Case Reports in Oncology}

\begin{tabular}{l|l}
\hline Case Rep Oncol 2014;7:804-809 \\
\hline DOI: 10.1159/000369994 & $\begin{array}{l}\text { ○ 2014 S. Karger AG, Basel } \\
\text { www.karger.com/cro }\end{array}$ \\
\hline
\end{tabular}

Furukawa et al.: Port-Site Metastasis of Mucinous Borderline Ovarian Tumor after Laparoscopy

6 Zivanovic 0, Sonoda Y, Diaz JP, et al: The rate of port-site metastases after 2,251 laparoscopic procedures in women with underlying malignant disease. Gynecol Oncol 2008;111:431-437.

7 Morice P, Uzan C, Fauvet R, Gouy S, Duvillard P, Darai E: Borderline ovarian tumour: pathological diagnostic dilemma and risk factors for invasive or lethal recurrence. Lancet Oncol 2012;13:e103-e115.

$>8$ Trimble CL, Kosary C, Trimble EL: Long-term survival and patterns of care in women with ovarian tumors of low malignant potential. Gynecol Oncol 2002;86:34-37.

-9 Uzan C, Nikpayam M, Ribassin-Majed L, et al: Influence of histological subtypes on the risk of an invasive recurrence in a large series of stage I borderline ovarian tumor including 191 conservative treatments. Ann Oncol 2014;25:1312-1319.
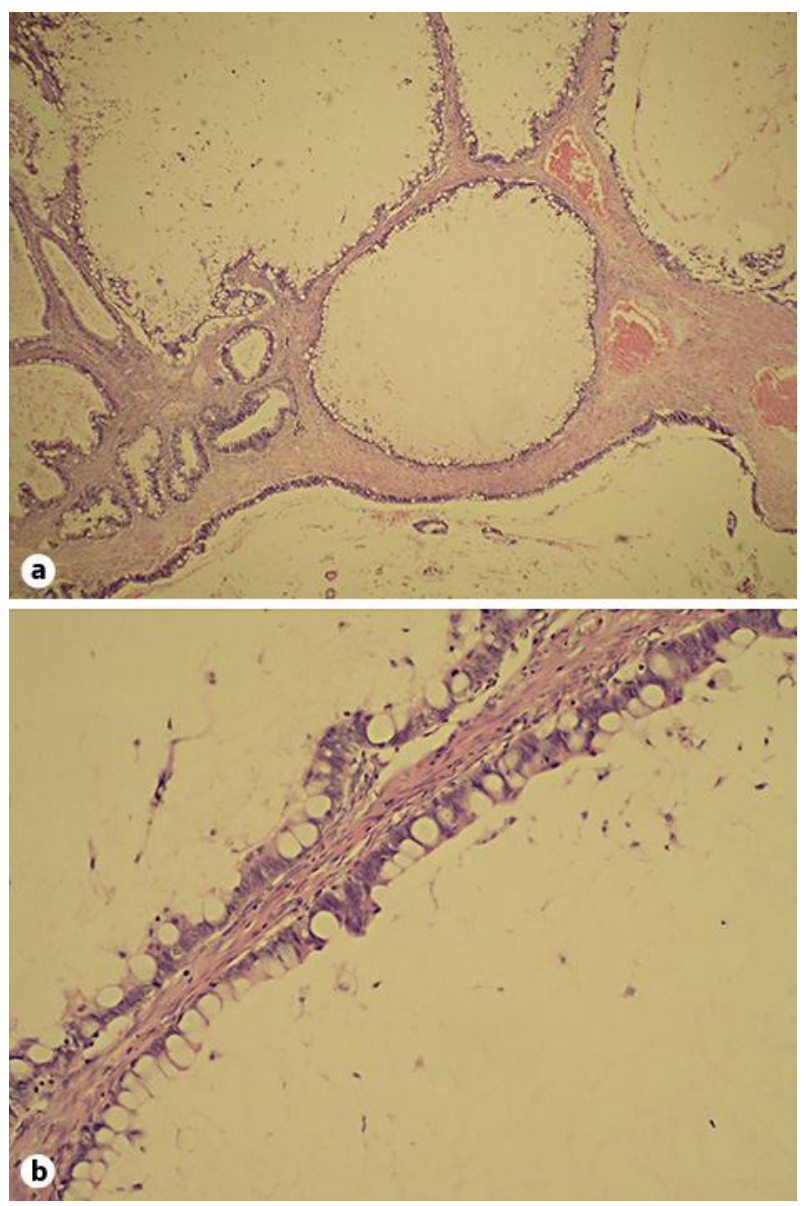

Fig. 1. Intestinal mucinous tumor showing numerous goblet cells with stratification and nuclear atypia. The epithelial-stromal junction is sharp and clear. a Hematoxylin and eosin $\times 40$; $\mathbf{b}$ hematoxylin and eosin $\times 400$. 
Furukawa et al.: Port-Site Metastasis of Mucinous Borderline Ovarian Tumor after Laparoscopy

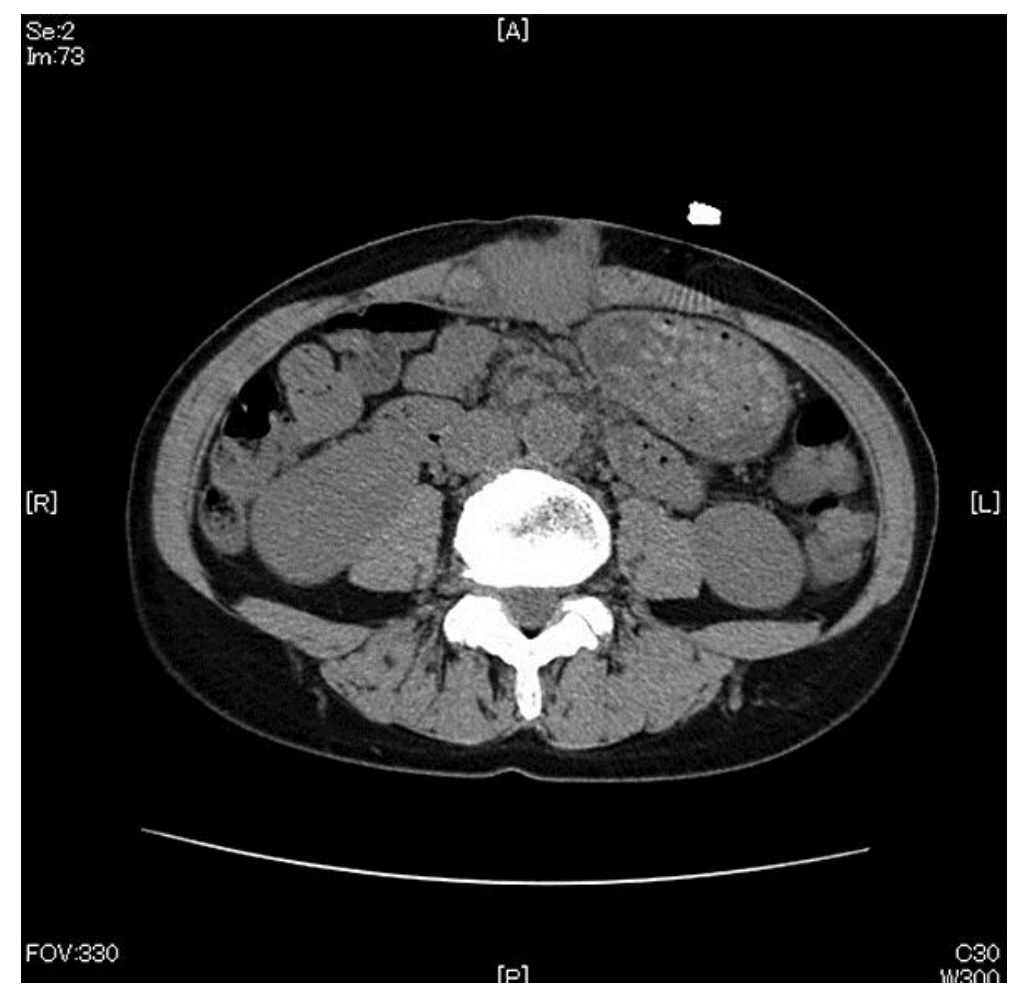

Fig. 2. Computed tomography scan showing a mass in the abdominal wall near the umbilicus. 


\section{Case Reports in Oncology}

\begin{tabular}{l|l}
\hline \multicolumn{2}{l}{ Case Rep Oncol 2014;7:804-809 } \\
\hline DOI: 10.1159/000369994 & $\begin{array}{l}\text { C 2014 S. Karger AG, Basel } \\
\text { www.karger.com/cro }\end{array}$ \\
\hline
\end{tabular}

Furukawa et al.: Port-Site Metastasis of Mucinous Borderline Ovarian Tumor after Laparoscopy
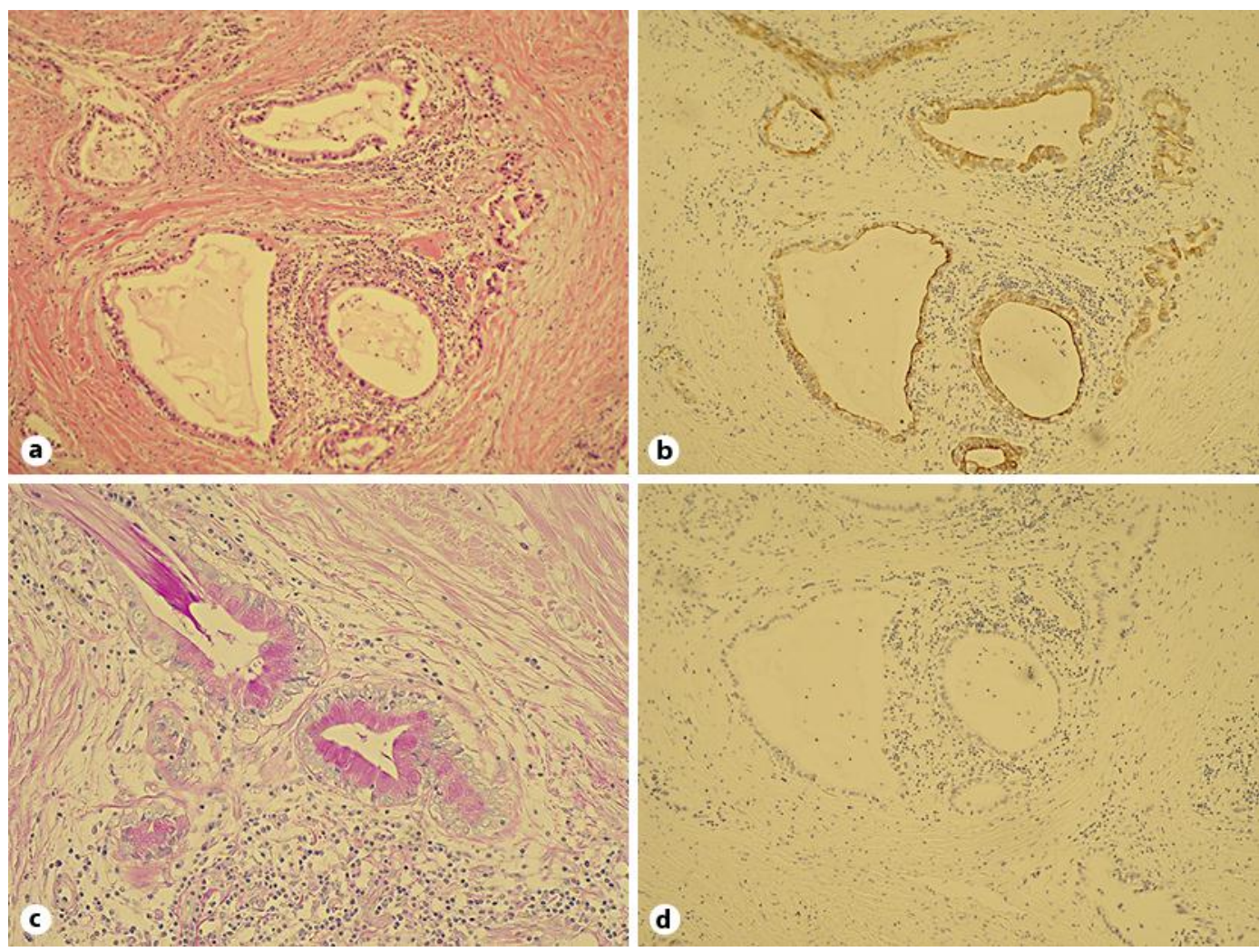

Fig. 3. a The mucinous tumor of the abdominal wall is infiltrating the stroma. $\mathbf{b}$ Tumor cells are positive for cytokeratin 7. c Tumor cells are positive for PAS. $\mathbf{d}$ Tumor cells are negative for cytokeratin 20. 